This is the peer-reviewed version of the following article: Cook RT \& Ebert PA (2016) Frege's Recipe, Journal of Philosophy, 113 (7), pp. 309-345, which has been published in final form at https://doi.org/10.5840/jphil2016113721. This article may be used for non-commercial purposes only.

\title{
Frege's Recipe
}

$\begin{array}{cc}\text { Roy T. Cook } & \text { Philip A. Ebert } \\ \text { Department of Philosophy } & \text { Division of Law and Philosophy } \\ \text { University of Minnesota } & \text { University of Stirling } \\ \text { Minneapolis, MN, } & \text { Stirling, Scotland, } \\ \text { USA } & \text { UK } \\ & \\ \text { cookx432@umn.edu.edu } & \text { p.a.ebert@stir.ac.uk }\end{array}$

February 23, 2016

\section{Introduction}

This paper has three aims: first, we present a formal recipe that Frege followed in his magnum opus Grundgesetze der Arithmetik ${ }^{1}$ when formulating definitions. This generalized recipe, as we will call it, is not explicitly mentioned as such by Frege, but we will offer strong reasons to believe that Frege applied the recipe in developing the formal material of Grundgesetze. Second, we will show that a version of Basic Law V plays a fundamental role in the generalized recipe. We will explicate exactly what this role is and how it differs from the role played by extensions in Die Grundlagen der Arithmetik. ${ }^{2}$ Third, and finally, we will demonstrate that this hitherto neglected yet foundational aspect of Frege's use of Basic Law V helps to resolve a number of important interpretative challenges in recent Frege scholarship, while also shedding light on some important differences between Frege's logicism and recent 'neo-logicist' approaches to the foundations of mathematics.

The structure of our paper is as follows: In the first section, we will outline Frege's semi-formal definition of cardinal numbers given in Grundlagen and present what we call the simple recipe. In the second section, we will outline two distinct ways to unpack the simple recipe formally, followed by a discussion of its philosophical and technical shortcomings. This leads naturally to the topic of the third section - the problem of the singleton - a problem that Frege was aware of and which, we believe, significantly shaped his views on definitions between Grundlagen and Grundgesetze. These observations motivate the introduction of the generalized recipe. In the

\footnotetext{
${ }^{1}$ Published in two volumes: (Frege, 1893) and (Frege, 1903), henceforth: Grundgesetze.

${ }^{2}$ (Frege, 1884), henceforth: Grundlagen.
} 
fourth section, we will explain this generalized modification of the simple recipe and demonstrate how it is applied in arriving at the majority of the definitions given in Grundgesetze. In the fifth section, we will argue that the generalized recipe has important philosophical consequences for Frege scholarship: we will sketch the beginnings of a new interpretation of the role and importance of Basic Law V and of Hume's Principle in Frege's mature (Grundgesetze-era) philosophy of mathematics. We close by noting some differences between Frege's project and the methodology of contemporary neo-logicism.

\section{Identifying abstracts in Grundlagen}

As is well known, in Grundlagen Frege rejected Hume's Principle: ${ }^{3}$

$$
H P:(\forall X)(\forall Y)\left[\phi_{\phi}(X)=\phi(Y) \leftrightarrow X \approx Y\right]
$$

as a definition of the concept CARDINAL NUmBer. ${ }^{4}$ Hume's Principle states that the number of $F$ 's is identical to the number of $G^{\prime} s$ if and only if the $F$ 's and the $G^{\prime} s$ are in one-to-one correspondence. Frege was very likely aware of the fact that Hume's Principle on its own (plus straightforward definitions of arithmetical concepts such as SUCCESSOR, ADDITION, and MULTIPLICATION) entails what we now call the second-order Dedekind-Peano axioms for arithmetic - a result that is known as Frege's Theorem. ${ }^{5}$ The reason for his rejection of Hume's Principle as a proper definition is known as the Caesar Problem:

... we can never - to take a crude example - decide by means of our definitions whether a concept has the number Julius Caesar

\footnotetext{
${ }^{3}$ Frege never used the expression "Hume's Principle". The use of this label is, however, entrenched amongst Frege scholars and so we will refer to this principle throughout using "Hume's Principle", even in a context when we discuss Frege's views about it.

$4 " q$ " is the cardinal-number operator, and " $X \approx Y$ " abbreviates the second-order formula stating that there is a one-to-one onto function from $X$ to $Y$. We shall partly translate Frege's Grundgesetze formulations into modern terminology - with appropriate comments regarding any theoretical mutilations that might result - but we will retain his original notation in quotations. Hence, we will use modern 'Australian' A's $(\forall)$ and 'backwards' E's $(\exists)$ for the quantifiers, and modern linear notation $(\rightarrow)$ for the material conditional. The reader should be aware that identity $(=)$ and equivalence $(\leftrightarrow)$ are, within Frege's Grundgesetze formalism, equivalent when the arguments are sentences (i.e. names of truth-values), and we will use whichever is more illuminating in our own formulations below.

${ }^{5}$ The label "Frege's Theorem" dates back to (Boolos, 1990). See also (Heck, 2011), chapter 1, p. 3ff on the historical context surrounding Frege's Theorem. (Dummett, 1991), p. 123 suggests that Frege was aware that Peano Arithmetic could be derived solely from Hume's Principle at the time of writing Grundlagen. However, as pointed out in (Boolos and Heck, 1998), Frege's sketch of this result - in particular, the proof sketch of the successor axiom-in Grundlagen is incorrect. Compare also (Heck, 2011), chapter 3.
} 
belonging to it, whether this famous conqueror of Gaul is a number or not.

$$
\text { (Frege, 1884), §56, p. } 68 \text {. }
$$

The worry, in short, is that an adequate definition of the concept CARDINAL NUMBER should settle all identities involving numerical terms, including those where the identity symbol "=" is flanked by a Fregean numeral (such as " $q_{(}(F)$ ") on one side and a non-numerical term (such as "Julius Caesar") on the other. Hume's Principle does not settle such identities and thus it is inadequate as a definition of the concept CARDINAL NUMBER.

Frege's proposed solution to the Caesar Problem is simple to state: in order to distinguish numbers from more 'pedestrian' objects, such as the conqueror of Gaul, Frege proposes that we identify cardinal numbers with certain extensions by means of an explicit definition. In Grundlagen, $\S 68$, immediately after a discussion of the Caesar Problem, Frege offers the following definition:

Accordingly, I define:

the cardinal number which belongs to the concept $F$ is the extension of the concept "equinumerous to the concept $F$ ".

(Frege, 1884), §68, p. 79-80.

Thus, cardinal numbers are a particular kind of extension. It is clear from his discussion in Grundlagen, however, that it is not just cardinal numbers but many (if not all) other mathematical objects that are to be identified with appropriate extensions. In the same section, he writes:

the direction of line $a$ is the extension of the concept "parallel to line $a "$

the shape of triangle $t$ is the extension of the concept "similar to the triangle $t$ ".

(Frege, 1884), §68, p. 79 .

The wide-ranging nature of these examples strongly suggests that Frege regarded this approach not merely as a technical fix to resolve particular cases involving Caesar-type examples, but rather as a codification of a basic insight into the nature of mathematical objects and mathematical concepts. Frege's identification of mathematical objects with the extension of corresponding equivalence classes amounts to a definitional method which seems generally applicable to all mathematical objects and concepts, including shapes, directions, and cardinal numbers. ${ }^{6}$ Hence, from this perspective

\footnotetext{
${ }^{6}$ There is, of course, Grundlagen $\S 107$, where Frege suggests that he attaches no particular importance to his use of the term "extensions of concepts". By the time of Grundgesetze, however, Frege attaches a great deal of importance to extensions of concepts,
} 
there is nothing special about cardinal numbers - they are just a particularly salient example of the definitional methodology applied in Grundlagen. ${ }^{7}$

Reflecting on Frege's methodology in Grundlagen, we obtain the following recipe for identifying the mathematical objects falling under some mathematical concept $C$ (such as DIRECTION or SHAPE), which we shall call the simple abstracta-as-extension recipe, or simply, the simple recipe: ${ }^{8}$

Step 1: Identify the underlying concept $\Phi_{C}$ such that $C$ 's are $C$ 's of $\Phi_{C}$ 's.

That is, if $C$ is the concept DIRECTION, then $\Phi_{C}$ is the concept Line, and if $C$ is the concept shaPe, then $\Phi_{C}$ is the concept TRIANGLE.

Step 2: Formulate the identity conditions for $C$ 's in terms of some appropriate equivalence relation $\Psi_{C}$ on the underlying domain of $\Phi_{C}$ 's.

That is, identify a formula of the form:

$$
\forall \phi_{1}, \phi_{2} \in \Phi_{C} \text {, the } C \text { of } \phi_{1}=\text { the } C \text { of } \phi_{2} \leftrightarrow \Psi_{C}\left(\phi_{1}, \phi_{2}\right)
$$

where $\Psi_{C}$ provides the identity conditions for $C$ 's. Thus, if $C$ is the concept DIRECTION, then $\Psi_{C}$ is the relation PARALLELISM, and if $C$ is the concept SHAPE, then $\Psi_{C}$ is the relation SIMILARITY.

Step 3: Identify the $C$ 's with the equivalence classes of relevant $\Phi_{C}$ 's (modulo the equivalence relation $\Psi_{C}$ ).

So, the direction of a line $\lambda$ is identified with the equivalence class of lines parallel to $\lambda$ :

$$
\operatorname{dir}(\lambda)=\dot{\varepsilon}(\varepsilon \| \lambda)
$$

and the shape of a triangle $\tau$ is identified with the equivalence class of triangles similar to $\tau$ :

$$
\operatorname{shp}(\tau)=\dot{\varepsilon}(\varepsilon \sim \tau)
$$

or more generally, value-ranges of functions. This reflects a deep change in Frege's views between the time of writing Grundlagen and Grundgesetze, one intimately connected to his abandoning the simple recipe in favor of the generalized recipe. More on this below.

${ }^{7}$ There is, of course, something special about cardinal numbers when compared to shapes and directions: cardinal numbers are defined as extensions of second-level concepts that hold of concepts (or, alternatively, of first-level concepts that hold of extensions of concepts). Thus, cardinal numbers, unlike shapes and directions, are logical objects since they are identified with equivalence classes of logical objects (either concepts or their extensions), while directions and shapes correspond to equivalence classes of non-logical objects (lines and geometrical regions respectively).

${ }^{8}$ Note that Frege does not seem to be giving a general account of the concept GEOMETRICAL SHAPE, but is instead providing a definition of the narrower concept SHAPE OF A TRIANGLE. Having noted this, however, we shall from here on ignore it since it is irrelevant to our present concerns. 
Step 4: Prove the relevant abstraction principle:

$$
\left(\forall \phi_{1}\right)\left(\forall \phi_{2}\right)\left[@_{C}\left(\phi_{1}\right)=@_{C}\left(\phi_{2}\right) \leftrightarrow \Phi_{C}\left(\phi_{1}, \phi_{2}\right)\right]
$$

where:

$$
@_{C}(\phi)=\dot{\varepsilon}\left(\Psi_{C}(\varepsilon, \phi)\right)
$$

Thus, given our definition identifying directions with equivalence classes of lines, we prove the adequacy of our definition of DIRECTION by proving:

$$
\left(\forall \lambda_{1}\right)\left(\forall \lambda_{2}\right)\left[\operatorname{dir}\left(\lambda_{1}\right)=\operatorname{dir}\left(\lambda_{2}\right) \leftrightarrow\left(\lambda_{1}|| \lambda_{2}\right)\right]
$$

that is:

$$
\left(\forall \lambda_{1}\right)\left(\forall \lambda_{2}\right)\left[\dot{\varepsilon}\left(\varepsilon \| \lambda_{1}\right)=\dot{\alpha}\left(\alpha \| \lambda_{2}\right) \leftrightarrow\left(\lambda_{1} \| \lambda_{2}\right)\right]
$$

and we prove the adequacy of our definition of SHAPE by proving:

$$
\left(\forall \tau_{1}\right)\left(\forall \tau_{2}\right)\left[\operatorname{shp}\left(\tau_{1}\right)=\operatorname{sh} p\left(\tau_{2}\right) \leftrightarrow\left(\tau_{1} \sim \tau_{2}\right)\right]
$$

that is:

$$
\left(\forall \tau_{1}\right)\left(\forall \tau_{2}\right)\left[\hat{\varepsilon}\left(\varepsilon \sim \tau_{1}\right)=\dot{\alpha}\left(\alpha \sim \tau_{2}\right) \leftrightarrow\left(\tau_{1} \sim \tau_{2}\right)\right]
$$

It is important to note that it is Step 3 that provides the definition. Step 4 amounts to proving that the given definition adequately captures the concept being defined: it functions as an adequacy constraint on the definition.

The examples just discussed are somewhat special since we have here applied the simple recipe only to first-order abstractions - that is, to definitions of mathematical concepts $C$ where the underlying $\Phi_{C}$ 's are objects and not second-(or higher-) order concepts, relations, or functions. The reason for this is that there is an apparent ambiguity in Frege's application of this construction to concepts, such as the concept CARDINAL NUMBER, whose underlying concept $\Phi_{C}$ is not objectual. We discuss this in the following section in more detail.

Another aspect in which the definitions of directions and shapes differ from the definition of cardinal numbers is that Frege does not explicitly carry out Step 4 of the simple recipe for directions or shapes, while he does so for cardinal numbers. ${ }^{9}$ After providing the definition in $\S 68$, and before sketching the derivation of a version of the Peano axioms in $\S 74-\S 83$, Frege has this to say:

We will first show that the cardinal number which belongs to the concept $F$ is equal to the cardinal number which belongs to the concept $G$ if the concept $\mathrm{F}$ is equinumerous to the concept $G$.

$$
\text { (Frege, 1884), §73, p } 85 .
$$

\footnotetext{
${ }^{9}$ Frege does, however, motivate these definitions of SHAPE and DIRECTION by appeal to their corresponding abstraction principles - see Grundlagen, $\S 68$. But unlike the case of cardinal numbers, he does not derive them.
} 
After sketching a proof of this claim - essentially, the right-to-left direction of Hume's Principle - Frege concludes the section with the following footnote:

And likewise of the converse: If the number which belongs to the concept $F$ is the same as that which belongs to the concept $G$, then the concept $F$ is equal to the concept $G{ }^{10}$

$$
\text { (Frege, 1884) } § 73, \text { p. } 86(\text { fn1). }
$$

Strictly speaking, then, Frege does not provide a full proof sketch of Hume's Principle in $\S 73$ of Grundlagen, but that he considers both directions in one section we regard as sufficient for our purposes. It is also noteworthy that the sections in which Frege sketches both a proof of (the two sides of) Hume's Principle and proofs of central principles of Peano Arithmetic fall under the heading "Our definition completed and its worth proved". Since these sections contain a derivation sketch of Hume's Principle first, and then show how to derive the more familiar arithmetic results from Hume's Principle, it seems natural to interpret the derivation of Hume's Principle as completing the definition and thus fulfil Step 4 of Frege's definitional strategy, while the derivation of the Peano axioms demonstrate the worth of the definition.

To summarise: in Grundlagen Frege provides two sorts of evidence that the definition of cardinal numbers as extensions is correct. He sketches a proof that the second-order Peano axioms follow from the definition (a task carried out with more rigor and in more detail in Grundgesetze). Yet, before engaging in the proof, he also notes that the definition entails (each direction of) Hume's Principle. In short, Frege carries out Step 4 of the simple recipe when applied to cardinal number and he thus regards Hume's Principle as providing a precise adequacy condition that any definition of the concept CARDINAL NUMBER must meet. This, in turn, explains the central role that Hume's Principle plays in Grundlagen despite being rejected as a definition proper.

\footnotetext{
${ }^{10}$ Similarly, in the concluding remarks of Grundlagen, Frege emphasizes the fact that any adequate definition of number must recapture (i.e. prove) the relevant principle governing recognition conditions, which in the case of cardinal numbers is Hume's Principle:

The possibility to correlate single-valuedly in both directions the objects falling under a concept $F$ with the objects falling under the concept $G$, was recognised as the content of a recognition-judgement for numbers. Our definition, therefore, had to present this possibility as co-referential (gleichbedeutend) with a numberequation. We here drew on similar cases: the definition of direction based on parallelism, of shape based on similarity.

(Frege, 1884), §106, p. 115.

After rehearsing the reasons for rejecting Hume's Principle itself as a definition in $\S 107$, Frege reminds us in $\S 108$ of his proof that the explicit definition of cardinal numbers in terms of extensions meets this criterion - that is, he reminds us of his proof of (the right-to-left direction of) Hume's Principle.
} 


\section{Two options for identifying abstracts}

In this section, we will outline two ways of unpacking Frege's identification of each number with 'the extension of the concept "equinumerous to the concept F"'. The first option involves understanding the cardinal number of $F$ as the extension of the (second-level) concept holding of those concepts equinumerous to $F$ - that is:

$$
\phi(F)=\dot{\varepsilon}(\varepsilon \approx F)
$$

Note that, on the first option, the extension operator $\dot{\varepsilon}$ binds a first-level concept variable, not an object variable. ${ }^{11}$

The second option involves understanding the cardinal number of $F$ as the extension of the (first-level) concept holding of the extensions of those concepts equinumerous to $F$ - that is:

$$
q_{q}(F)=\dot{\varepsilon}((\exists Y)(\varepsilon=\dot{\alpha}(Y(\alpha)) \wedge Y \approx F))
$$

Although this ambiguity is cleared up in the formal treatment of arithmetic in Grundgesetze, we will consider both proposals suggested by the looser presentation in Grundlagen. Such an approach will illustrate that, in applying the recipe the choice between the first option and the second option is not arbitrary or merely a matter of convenience. Instead, there are principled reasons for defining cardinal numbers - and, more generally, all secondorder abstracts - as extensions of first-level concepts holding of extensions of concepts. Thus, there are good reasons for Frege - reasons we believe he was aware of - to adopt the second option.

\subsection{The first option}

The first way of understanding Frege's suggestion that the cardinal number of the concept $F$ is the extension of the concept EQUINUMEROUS TO THE CONCEPT $F$ is to identify the number of $F$ with the extension of the secondlevel concept holding of all first-level concepts equinumerous to $F$ :

$$
\phi(F)=\dot{\varepsilon}(\varepsilon \approx F)
$$

If this were the right way to understand Frege, then we can generalise the simple recipe to higher-level concepts. Given any mathematical concept

\footnotetext{
${ }^{11}$ Frege utilizes extensions of concepts within Grundlagen, while mobilizing the more general notion of value-ranges of functions within Grundgesetze. Extensions of concepts, however, are a special kind of value-range: they are the value-ranges of unary concepts, where concepts are functions whose range is the True and the False. Since we shall be shifting frequently between Frege's Grundlagen definition of cardinal number and his Grundgesetze definition of the cardinal number, we shall use the Grundgesetze notation " $\dot{\varepsilon}(\ldots \varepsilon \ldots)$ " throughout, taking care to flag when the broader notion of value-range, rather than extension, is at issue.
} 
$C$, with associated underlying (second-level) concept $\Phi_{C}$ and (second-level) equivalence relation $\Psi_{C}$, we can identify the $C$ 's as follows:

$$
@_{C}(F)=\dot{\varepsilon}\left(\Psi_{C}(\varepsilon, F)\right)
$$

where $@_{C}$ is the abstraction operator mapping concepts to $C$ 's.

In particular, applying the first option to extensions themselves provides:

$$
\dot{\alpha}(F(\alpha))=\dot{\varepsilon}((\forall y)(F(y) \leftrightarrow \varepsilon(y)))
$$

This substitution will be admissible if the recipe is not only a means for identifying 'new' objects (or, more carefully: for defining new concepts by identifying which of the 'old' objects - the extensions - fall under those concepts) but it is, more generally, a method for identifying any mathematical objects.

There are, we think, good reasons for interpreting the recipe in the broader sense: the definitional strategy adopted by Frege in Grundlagen is not merely intended to identify which objects are the cardinal numbers, but it is intended to play a more general role in Frege's logicism. In order to gain epistemological access to some objects falling under a mathematical concept $C$, a definition has to provide us with identity conditions for the objects falling under $C$. If the recipe achieves this for mathematical objects falling under a mathematical concept $C$ via an identification of the objects falling under $C$ with particular extensions (and hence applies to at least these extensions), then it should apply to all extensions, including those objects that do not fall under one or another mathematical/logical concept other than EXTENSION itself. Otherwise, the domain of extensions would be artificially partitioned into two sub-domains corresponding to distinct means for determining identity conditions: those extensions that fall in the range of a mathematical concept other than EXTENSION to which the recipe applies, and those that do not. ${ }^{12}$

For this reason, we think that Frege's recipe should also apply to extensions themselves. ${ }^{13}$ In that case, however, the first option reading of the simple recipe must be rejected as the proper understanding of Frege's method for defining mathematical concepts and identifying the corresponding objects. The reason is simple: The first option is logically incoherent. If we identify

\footnotetext{
${ }^{12}$ The point is not that, on the simple recipe, identity conditions for cardinal numbers are not given in terms of Basic Law V. If cardinal numbers are, in fact, extensions, then they can be individuated using Basic Law V just like any other extension. The point is that the philosophically primary identity criterion for cardinal numbers is equinumerosity, which must then be analyzed in terms of the recipe to reduce it to a relation on relevant extensions.

${ }^{13}$ Note that we do not need the (implausible) claim that this reading of the simple recipe provides us with a definition of extensions, but rather the weaker claim that applying the simple recipe to value-ranges (which, for Frege, require no definition) results, not in a definition, but in a truth.
} 
the extension of a first-level concept with the extension of a second-level concept, then we need some general means for settling such cross-level identity statements. According to Basic Law V, however, extensions of concepts can only be identical when the concepts in question hold of exactly the same thing or things. No first-level concept can hold of anything that any second-level concept holds of, since first-level concepts hold of objects and second-level concepts hold of first-level concepts. As a result (and assuming that we extend the notion of extension to second-level concepts in the first place) the only logically possible pair $\left\langle C_{1}, C_{2}\right\rangle$ where $C_{1}$ is a first-level concept, $C_{2}$ is a second-level concept, and $\dot{\varepsilon}\left(C_{1}(\varepsilon)\right)=\dot{\varepsilon}\left(C_{2}(\varepsilon)\right)$ is the case where $C_{1}$ and $C_{2}$ are both empty concepts (although obviously not the 'same' empty concept, since they are of different levels). As a result, the first option is not a live option. In particular, the identity in question:

$$
\dot{\alpha}(F(\alpha))=\dot{\varepsilon}((\forall y)(F(y) \leftrightarrow \varepsilon(y)))
$$

must, at best, always be false, since the degenerate case where both $F$ and $(\forall y)(F(y) \leftrightarrow X(y))$ hold of nothing whatsoever is not possible here: $(\forall y)(F(y) \leftrightarrow X(y))$ holds of $F$.

Now, once Frege had formulated the logic of Grundgesetze in the required detail, he would have, no doubt, realised that the first option does not apply to extensions (or value-ranges for that matter). If, as we argued above, Frege's recipe has to apply to all mathematical objects, then the failure of the first option can now be interpreted in the wider context of motivating a shift from the first to the second option. Thus, in contrast to other interpreters, we think that Frege's adoption of the second option in Grundgesetze is not merely a choice based on convenience but it is a well-motivated move to fulfil the requirements of his recipe. ${ }^{14}$

\subsection{The Second Option}

The second way of understanding Frege's suggestion that the number of the concept $F$ is the extension of the concept EQUINUMEROUS TO THE CONCEPT $F$ is to identify the number of $F$ with the extension of the first-level concept holding of the extensions of all first-level concepts equinumerous to $F$ :

$$
\phi(F)=\dot{\varepsilon}((\exists Y)(\varepsilon=\dot{\alpha}(Y(\alpha)) \wedge Y \approx F)
$$

In order to simplify our presentation, we will now incorporate one of Frege's own tricks: Frege does not define equinumerosity as a second-level relation holding of pairs of first-level relations, but instead defines it as a

\footnotetext{
${ }^{14}$ Consider e.g. (Blanchette, 2012) who interprets Frege's move from the first to the second option as "simply [...] one of technical convenience" (p. 83). We pick up on the issue of arbitrariness in section 4.1. Further discussion of Blanchettes interpretation of this issue can be found in (Cook, 2015).
} 
first-level relation holding of the extensions of first-level concepts. Hence, " $\alpha \approx \beta$ " is true if and only if $\alpha$ and $\beta$ are the extensions of (first-level) concepts $F_{\alpha}$ and $F_{\beta}$ such that the $F_{\alpha}$ s are equinumerous to the $F_{\beta}$ s. ${ }^{15}$

With this new understanding of " $\approx$ " in place, Frege's definition of cardinal numbers becomes:

$$
\phi_{\phi}(F)=\dot{\varepsilon}(\varepsilon \approx \dot{\alpha}(F(\alpha))
$$

This is, essentially, the definition of number provided by Frege in Grundgesetze. ${ }^{16}$

As was the case with the first option, Frege's application of the second option version of the simple recipe to the concept CARDINAL NUMBER can be straightforwardly generalized so as to be applicable to abstracts of any first-level concepts. Given any mathematical concept $C$, with associated underlying (second-level) concept $\Phi_{C}$ and (second-level) equivalence relation $\Psi_{C}$, we can identify the $C$ 's as follows:

$$
@_{C}(F)=\dot{\varepsilon}\left((\exists Y)\left(\varepsilon=\S(Y) \wedge \Psi_{C}(Y, F)\right)\right)
$$

where $@_{C}$ is the abstraction operator mapping concepts to $C$ 's. Importantly, the second option version of the simple recipe does not result in logical incoherence and it is thus an improvement on the first option.

Nonetheless, the simple recipe does have its limitations: first, the fact that the second option depends on identifying abstract objects via equivalence relations restricts its applicability to unary abstracts, and hence does not apply to concepts $C$ where the abstracts falling under $C$ result from abstracting off more than one of the underlying $\Phi_{C}$ 's - a problem that would,

\footnotetext{
${ }^{15}$ Strictly speaking, Frege does not explicitly define equinumerosity in Grundgesetze, but instead defines a 'mapping into' operation. His official definition of number involves a complicated formula involving a complex subcomponent equivalent to equinumerosity, constructed in terms of the 'mapping into' construct. The lack of an explicit definition of equinumerosity in Grundgesetze further emphasizes a fact that we will bring out later: that Hume's Principle plays no role in the formal development of Grundgesetze.

${ }^{16}$ There is a difference between the definition of cardinal number that results from this reading of the simple recipe and the superficially similar formal definition given in Grundgesetze: Within Grundgesetze Frege's definition of equinumerosity implies that two functions $\mathcal{F}_{1}$ and $\mathcal{F}_{2}$ are equinumerous if and only if there is a one-one onto mapping between the arguments that $\mathcal{F}_{1}$ maps to the True and the arguments that $\mathcal{F}_{2}$ maps to the True, regardless of whether these functions map all other arguments to the False (that is, regardless of whether these functions are concepts). Thus, the mature Grundgesetze definition of cardinal number does not identify numbers with 'collections' of extensions of equinumerous concepts, but rather with 'collections' of value-ranges of functions (including but not restricted to concepts) that map equinumerous collections of objects to the True. Along similar lines, the ordered pair of $\alpha$ and $\beta$, which shall be examined in detail below, is the 'collection' of (the double value-ranges of) all functions that map $\alpha$ and $\beta$ (in that order) to the True, and not the (less-inclusive) collection of (value-ranges of) relations that relate $\alpha$ to $\beta$. This observation, while important for other reasons, is orthogonal to our concerns in this paper, so we ignore it. For further discussion of the issue, see (Cook, 2014).
} 
of course, also affect the first option. So, for example, the simple recipe will not provide us with a pairing operation (more on this below).

Second, and more importantly at this stage, the simple recipe gets identity conditions wrong in specific cases. And here, once again, the problem is to apply the recipe to extensions. Now, while the second option of applying the simple recipe does not result in a logical incoherence, we do, however, face what we call the problem of the singleton. This problem arises when we apply the second option understanding of the simple recipe to extensions themselves, obtaining:

$$
\dot{\varepsilon}(F(\varepsilon))=\dot{\alpha}((\exists X)(\alpha=\dot{\varepsilon}(X(\varepsilon)) \wedge(\forall y)(F(y) \leftrightarrow X(y)))
$$

This is equivalent (modulo Basic Law V) to:

$$
\dot{\varepsilon}(F(\varepsilon))=\dot{\alpha}(\alpha=\dot{\varepsilon}(F(\varepsilon)))
$$

In short, applying this variant of the simple recipe to extensions themselves entails (using slightly anachronistic terminology) that every extension is identical to its singleton. This result, however, is problematic. If we instantiate the formula above with the empty concept $C_{\emptyset}$ :

$$
\dot{\varepsilon}\left(C_{\emptyset}(\varepsilon)\right)=\dot{\alpha}\left(\alpha=\dot{\varepsilon}\left(C_{\emptyset}(\varepsilon)\right)\right)
$$

Basic Law V entails that the empty extension $\dot{\varepsilon}\left(C_{\emptyset}(\varepsilon)\right)$ and any singleton extension are individuated extensionally, hence:

$$
(\forall x)\left(C_{\emptyset}(x) \leftrightarrow x=\dot{\varepsilon}\left(C_{\emptyset}(\varepsilon)\right)\right)
$$

Since the empty concept $C_{\emptyset}$ holds of no object, we obtain:

$$
(\forall x)\left(x \neq \dot{\varepsilon}\left(C_{\emptyset}(\varepsilon)\right)\right)
$$

and hence the contradiction:

$$
\dot{\varepsilon}\left(C_{\emptyset}(\varepsilon)\right) \neq \dot{\varepsilon}\left(C_{\emptyset}(\varepsilon)\right)
$$

It is worth emphasizing that the problem of the singleton does not depend in any way on the paradoxical character of Basic Law V itself. So long as we accept that the empty extension exists, that the simple recipe applies to extensions, and that identity conditions for abstracts are governed by some abstraction principle that settle the identity of the empty extension and of singletons in terms of co-extensionality (even if it disagrees with Basic Law $\mathrm{V}$ elsewhere), then the problem will arise. ${ }^{17}$

\footnotetext{
${ }^{17}$ In particular, the problem of the singleton would still be a problem in formal systems that replace the inconsistent Basic Law $\mathrm{V}$ with any of the consistent restricted versions of it explored in recent neo-logicist literature, such as George Boolos' NewV in (Boolos, 1989).
} 
So to summarise: while the second option is, in some way, an improvement on the first option for identifying abstracts using the simple recipe, it also fails as a general recipe for providing identity conditions for all mathematical objects. It fails in its application to extensions themselves and it does not easily generalise to non-unary abstracts. All this suggests that the simple recipe itself is in need of some revisions so to be better-suited for the purposes of Frege's logicism as defended in Grundgesetze. In section 4, we will show that the definitions Frege gives in Grundgesetze follow a modified generalized recipe.

Before outlining the new recipe, however, we should ask whether there are good reasons for thinking that Frege was aware of the problems affecting his simple recipe, and whether there are good grounds for thinking that he rejected it for the reasons we have offered. In the next section, we will show that Frege was familiar with a version of the problem of the singleton by the time of Grundgesetze. This, in turn, provides some evidence that his shift from the simple recipe of Grundlagen to the generalized recipe of Grundgesetze was quite possibly motivated, in part, by the kinds of concerns we have discussed above.

\section{$3 \quad$ Frege on singletons}

The most straightforward explanation of the problem of the singleton is that it is brought about by the commitment to identifying extensions and their singletons - a commitment implicitly codified in the simple recipe. Identifying objects with their singletons generally is implausible at best. ${ }^{18}$ Frege himself was aware of the danger of such an identification and discusses it near the end of $\S 10$ of Grundgesetze, vol. I.

Given that in Grundgesetze sentences are names of truth-values, the logic of Grundgesetze involves, at a glance, reference to two distinct types of logical object: truth-values and value-ranges. In order to reduce the number of types of logical objects - with a view to settling all identities within Grundgesetze in terms of identity conditions for value-ranges as codified in Basic Law $\mathrm{V}$-Frege makes two stipulations. First, he stipulates that the reference of true sentences - the True - is to be identified with the extension of any concept that holds of exactly the True. In short, the True is identical to the singleton of the True:

$$
\text { The True }=(\forall x)(x=x)=\dot{\varepsilon}(\varepsilon=(\forall x)(x=x))
$$

Second, he stipulates that the False is identical to the the singleton of the False:

$$
\text { The False }=(\forall x)(x \neq x)=\dot{\varepsilon}(\varepsilon=(\forall x)(x \neq x))
$$

\footnotetext{
${ }^{18}$ This is true even though identifying Urelemente- - that is, non-sets - with their singletons is often convenient and sometimes desirable.
} 
He follows up this observation with the following (rather hefty) footnote:

It suggests itself to generalise our stipulation so that every object is conceived as a value-range, namely, as the extension of a concept under which it falls as the only object. A concept under which only the object $\Delta$ falls is $\Delta=\xi$. We attempt the stipulation: let $\dot{\varepsilon}(\Delta=\varepsilon)$ be the same as $\Delta$. Such a stipulation is possible for every object that is given to us independently of value-ranges, for the same reason that we have seen for truth-values. But before we may generalise this stipulation, the question arises whether it is not in contradiction with our criterion for recognising valueranges if we take an object for $\Delta$ which is already given to us as a value-range. It is out of the question to allow it to hold only for such objects which are not given to us as value-ranges, because the way an object is given must not be regarded as its immutable property, since the same object can be given in different ways. Thus, if we insert ' $\alpha \Phi(\alpha)$ ' for ' $\Delta$ ' we obtain

$$
\text { ' } \dot{\varepsilon}(\dot{\alpha} \Phi(\alpha)=\varepsilon)=\dot{\alpha} \Phi(\alpha) \text { ' }
$$

and this would be co-referential with

$$
\text { ' } \mathfrak{a}-(\dot{\alpha} \Phi(\alpha)=\mathfrak{a})=\Phi(\mathfrak{a}),,
$$

which, however, only refers to the True, if $\Phi(\xi)$ is a concept under which only a single object falls, namely $\dot{\alpha} \Phi(\alpha)$. Since this is not necessary, our stipulation cannot be upheld in its generality.

The equation ' $\dot{\varepsilon}(\Delta=\varepsilon)=\Delta$ ' with which we attempted this stipulation, is a special case of ' $\varepsilon \Omega(\varepsilon, \Delta)=\Delta^{\prime}$ ', and one can ask how the function $\Omega(\xi, \zeta)$ would have to be constituted, so that it could generally be specified that $\Delta$ be the same as $\dot{\varepsilon} \Omega(\varepsilon, \Delta)$. Then

$$
\dot{\varepsilon} \Omega(\varepsilon, \dot{\alpha} \Phi(\alpha))=\dot{\alpha} \Phi(\alpha)
$$

also has to be the True, and thus also

$$
\text { a } \Omega(\mathfrak{a}, \dot{\alpha} \Phi(\alpha))=\Phi(\mathfrak{a}),
$$

no matter what function $\Phi(\xi)$ might be. We shall later be acquainted with a function having this property in $\xi \cap \zeta$; however we shall define it with the aid of the value-range, so that it cannot be of use for us here.

(Frege, 1893), §10, p. 18.

There are a few things worth noting regarding this passage. First, Frege is clearly aware that we cannot in general identify extensions with their singletons, noting that doing so results in identities of the form:

$$
\dot{\varepsilon}(F(\varepsilon))=\dot{\alpha}(\alpha=\dot{\varepsilon}(F(\varepsilon)))
$$


Such identities can only hold when the concept $F$ holds of exactly one object, since this formula entails that $F$ holds of exactly $\dot{\varepsilon}(F(\varepsilon))$. This is, in essence, the same point made above: we assumed that $F$ held of no objects, and then derived a contradiction. A similar reductio ad absurdum can of course be performed if we assume that $F$ holds of more than one object (and we assume that both the extension of $F$ and singletons are individuated in terms of co-extensionality).

Crucially, Frege does more than just point out that the identification of extensions with their singletons fails. In addition, he asks whether there is a relation $R$ such that:

$$
\dot{\varepsilon}(F(\varepsilon))=\dot{\alpha}(R(\alpha, \dot{\varepsilon}(F(\varepsilon))))
$$

does, in fact, hold generally. As we have already seen, identity is not such a relation, but it is open - as of $\S 10$ of Grundgesetze - whether there is some other relation $R$ such that the extension of a concept $F$ is identical to the extension of the concept "is $R$-related to $\dot{\alpha}(F(\alpha))$ ". For any such $R$, it must be the case that:

$$
(\forall x)(F(x) \leftrightarrow R(x, \dot{\varepsilon}(F(\epsilon))))
$$

holds. He then points out that his application operator " $\cap$ ", which we shall return to below, satisfies this constraint.

What is obvious from all of this is that Frege has a deep understanding of the perils that came with identifying extensions with their singletons. But, no one was (or likely is) more knowledgable about the technical intricacies of the formal system of Grundgesetze and their philosophical implications than Frege. Hence, it seems very unlikely that he would not have realized the consequences the problem of the singleton has for his earlier definitional strategy by the time of Grundgesetze.

\section{Identifying Abstracts in Grundgesetze}

Frege's Grundlagen definitions (that is, the results of applying the simple recipe using the second option), as well as almost all of Frege's Grundgesetze definitions, can be seen as instances of a more general method: the generalized recipe. With a single exception - the definition of the application operator " $\curvearrowright$ ", which we will return to later-Frege's definitions in Grundgesetze fall into three categories:

First, there are definitions of particular singular terms, such as zero "Q" (definition $\Theta$ ), one "1" (definition I), Endlos " $\varpi$ " (definition M), and definitions of particular relation symbols such as the successor relation $f$ (definition $\mathrm{H}$ ). With respect to the latter (and other particular relations defined later in Grundgesetze), Frege does not provide a definition of the successor relation in the modern sense, but rather identifies the object that 
is the double value-range of the relation in question. Hence, these definitions are all straightforward identifications of specific objects - in particular, of specific single or double value-ranges.

Second, there are definitions of what modern readers would naturally think of as (open or 'unsaturated') function or relation symbols, but which Frege formalized as (double value-ranges of) functions from value-ranges to value-ranges. In addition to the cardinal number operation discussed above (a function from concepts to extensions), these include basic operations on relations, including the composition of relations $p$ and $q$ (Definition $\mathrm{B}$ ):

$$
\Vdash \dot{\alpha} \dot{\varepsilon}\left(\pi^{\mathfrak{r}}\left[\begin{array}{r}
\varepsilon \cap(\mathfrak{r} \cap p) \\
\mathfrak{r} \cap(\alpha \frown q)
\end{array}\right)=p \sqcup q\right.
$$

the converse of a relation $p$ (Definition $\mathrm{E}$ ):

$$
\Vdash \dot{\alpha} \dot{\varepsilon}(\alpha \cap(\varepsilon \cap p))=\$ p
$$

and the coupling of relations $p$ and $q$ (Definition $\mathrm{O}$ ):

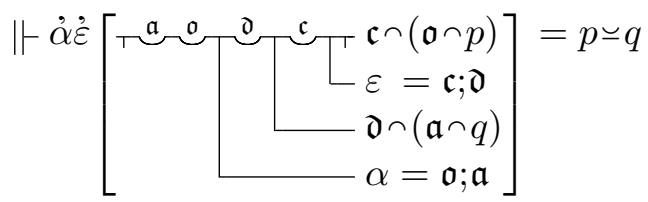

Each of these definitions identifies a function that takes objects (including double value-ranges of relations) as arguments, and provides the double value-range of another relation as value.

Third, we have definitions of what modern readers would naturally identify as predicates, but which Frege takes to be function symbols designating functions from objects (again, including single or double value-ranges) to truth-values. The first example of such a 'predicate' is Frege's definition $\Gamma$ :

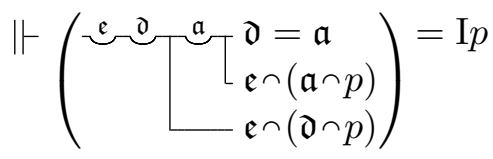

- the definition of the single-valuedness of a relation. Given any particular double value-range $p$ as argument, this expression denotes a truth-value: ${ }^{19}$

\footnotetext{
${ }^{19}$ Of course, as we have already seen, Frege in Grundgesetze identifies truth-values with value-ranges - in particular, with their own singletons. Thus, Frege's definitions of 'predicates' such as "I" are, in fact, functions from value-ranges to value-ranges. Since Frege's identification of truth-values with their singletons is never codified in an official basic law, however, but occurs instead in 'unofficial' philosophical discussion of the formalism (see Grundgesetze $\S 10$, vol. I), the wording given above is preferred.

The point - that is, the real distinction between Frege's treatment of operations on relations such as "ऽ", "\&", and "ㄷ", and his treatment of "predicates' such as "I"-is
} 
the True if the relation is single-valued - that is, if it is a function-and the False if it is not.

So how does Frege arrive at these particular definitions, and why do they fall into these three categories? These definitions follow from an application of the generalized recipe, which can be rationally reconstructed as follows:

Step 1: Identify the underlying concept $\Phi_{C}$ such that $C$ 's are $C$ 's of $\Phi_{C}$ 's. ${ }^{20}$

Step 2: Formulate the identity conditions for $C$ 's in terms of some appropriate relation $\Psi_{C}$ on the underlying domain of $\Phi_{C}$ 's such that:

$$
\begin{aligned}
& \forall \phi_{1}, \ldots \phi_{n}, \phi_{n+1}, \ldots \phi_{2 n} \in \Phi_{C} \\
& {\left[C\left(\phi_{1}, \ldots \phi_{n}\right)=C\left(\phi_{n+1}, \ldots \phi_{2 n}\right) \leftrightarrow \Psi_{C}\left(\phi_{1}, \ldots \phi_{n}, \phi_{n+1}, \ldots \phi_{2 n}\right)\right]}
\end{aligned}
$$

Step 2.5: Via applications of Basic Law V, transform the righthand-side of the biconditional into an identity:

$$
\Psi_{C}\left(\phi_{1}, \ldots \phi_{n}, \phi_{n+1}, \ldots \phi_{2 n}\right) \leftrightarrow f_{C}\left(\phi_{1}, \ldots \phi_{n}\right)=f_{C}\left(\phi_{n+1}, \ldots \phi_{2 n}\right)
$$

Step 3: Identify the $C$ 's with the range of $f_{C}$. In particular:

$$
C\left(\phi_{1}, \ldots \phi_{n}\right)=f_{C}\left(\phi_{1}, \ldots \phi_{n}\right)
$$

The generalized recipe involves two modifications to the simple recipe. The first is replacing Step $\mathbf{2}$ in the former with a more general and flexible two-step process (Step 2 and Step 2.5). The second modification is the deletion of Step 4. These modifications are both natural and necessary in order to carry out the work Frege wishes to carry out in Grundgesetze. In the following, we will illustrate how the first modification is essential in capturing Frege's Grundgesetze definitions and explore some technical and

that he did not define the latter as the extension of the concept holding of exactly those objects satisfying the predicate. In short, he did not define "I" as:

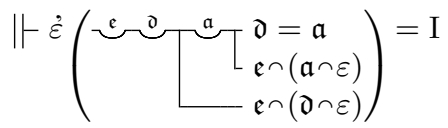

parallel to his definitions of "ऽ", " $\&$ ", and "ㄷ", and then write " $p \wedge I$ " instead of "I $p$ ". The

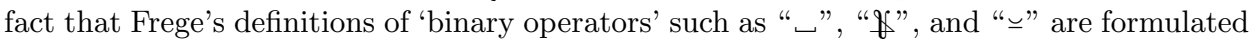
as functions from pairs of objects to double value-ranges (which are not truth-values, even on Frege's identification of truth-values with their singletons), while unary function symbols (i.e. 'predicates') such as "I" are defined as functions from objects to truth-values, deserves further scrutiny.

${ }^{20} \mathrm{By}$ the time of Grundgesetze, for Frege the underlying $\Phi_{C}$ s are always some class of objects, including single- and double value-ranges. Thus, numbers are, at least in a technical sense, not directly numbers of first-level concepts, but are instead numbers of the extensions of first-level concepts. 
philosophical consequences of this fact. Then, after three short digressions, we will conclude the paper by examining the second modification to the recipe, i.e. abolishing Step 4.

When applying the generalized recipe to unary operations that provide us access to numbers, directions, and shapes, the result is equivalent to that obtained when applying the simple recipe, although the details involved in getting to this final result are sometimes different. ${ }^{21}$ For example, letting our concept $\mathrm{C}$ be CARDINAL NUMBER, the underlying $\Phi_{C}$ is just the first-level concept extension of A FIRST-Level COnCEPt. At Step 2 we note that cardinal numbers are individuated in terms of equinumerousity - that is:

$$
\left(\forall \phi_{1}\right)\left(\forall \phi_{2}\right)\left[\phi\left(\phi_{1}\right)=\phi\left(\phi_{2}\right) \leftrightarrow \phi_{1} \approx \phi_{2}\right]
$$

Note that the number operator $\$$ now attaches, not to concepts, but to objects - that is, $\phi_{1}$ and $\phi_{2}$ are now first-order variables (further, we are again utilizing Frege's understanding of equinumerousity as a relation between extensions of concepts). ${ }^{22}$ We then note that the right-hand side is equivalent to:

$$
(\forall z)\left(z \approx \phi_{1} \leftrightarrow z \approx \phi_{2}\right)
$$

which, via Basic Law V (and some straightforward logical manipulation) is equivalent to:

$$
\dot{\alpha}\left(\alpha \approx \phi_{1}\right)=\dot{\alpha}\left(\alpha \approx \phi_{2}\right)
$$

Hence, on the generalized recipe, the cardinal number of $x$, for any object $x$, is the equivalence class of extensions of concepts equinumerous to $x$ :

$$
\phi(x)=\dot{\alpha}(\alpha \approx x)
$$

If $x$ is the extension of a concept:

$$
x=\dot{\varepsilon}(F(\varepsilon))
$$

however, then on the Grundgesetze account $\phi_{(}(x)$ will (speaking a bit loosely) pick out the same extension as $q_{(}(F)$ picked out on the Grundlagen simple recipe account.

We can also apply the generalized recipe to arrive at Frege's definition of the pairing operation ";" (Definition $\Xi$ ). The concept $C$ in question is (ordered) pair. The underlying concept $\Phi_{C}$ such that $C$ 's are $C$ 's of $\Phi_{C}$ 's is the concept OBJECT (Step 1). Things get a bit trickier at Step 2 and Step 2.5, however, since we are no longer looking for an equivalence relation on objects, but an 'equivalence relation'-like four-place relation. For

\footnotetext{
${ }^{21}$ In particular, and unlike the simple recipe, on the generalized recipe all definitions will take objects - usually but not necessarily extensions of concepts - as arguments.

${ }^{22}$ Note that, as a result, $\phi$ is defined for all objects, but it need only be 'well-behaved' in the intended case, where " $x$ " is the extension of a concept.
} 
the contemporary reader, with a century of sophisticated set theory under her belt, the appropriate relation with which to begin is obvious - pairwise identity:

$$
\forall \phi_{1}, \phi_{2}, \phi_{3}, \phi_{4} \in \Phi_{C}\left[\phi_{1} ; \phi_{2}=\phi_{3} ; \phi_{4} \leftrightarrow\left(\phi_{1}=\phi_{3} \wedge \phi_{2}=\phi_{4}\right)\right]
$$

We now note that the right-hand-side is equivalent to: ${ }^{23}$

$$
(\forall R)\left(R\left(\phi_{1}, \phi_{2}\right) \leftrightarrow R\left(\phi_{3}, \phi_{4}\right)\right)
$$

which is in turn equivalent to: ${ }^{24}$

$$
\left.\forall R\left(\phi_{1} \cap\left(\phi_{2} \cap \dot{\varepsilon} \dot{\alpha}(R \varepsilon \alpha)\right)=\phi_{3}\right\urcorner\left(\phi_{4} \cap \dot{\varepsilon} \dot{\alpha}(R \varepsilon \alpha)\right)\right)
$$

which, again via Basic Law V, becomes:

$$
\dot{\varepsilon}\left(\phi_{1} \cap\left(\phi_{2} \cap \varepsilon\right)\right)=\dot{\varepsilon}\left(\phi_{3} \cap\left(\phi_{4} \cap \varepsilon\right)\right)
$$

We now have the required identity, and can apply Step 3:

$$
x ; y=\dot{\varepsilon}(x \cap(y \cap \varepsilon))
$$

and we arrive at Frege's definition of ordered pair. ${ }^{25}$

In order to justify our claim that all of Frege's Grundgesetze definitions (with the exception of " $\cap$ ") flow naturally from the generalized recipe, it is worth working though a different example - the definition of the singlevaluedness of a function (Definition $\Gamma$ ). This function maps double valueranges of relations to truth-values, so the underlying concept $\Phi_{C}$ is just DOUble VAlue-RANGe. Equally straightforward is the application of Step $\mathbf{2}$-formulating the identity conditions. Since "I" is the sign of a function from objects to truth-values, determining the identity conditions for I just amounts to determining which arguments are mapped to the True, and which arguments are mapped to the False. Hence:

$$
\begin{aligned}
& \left(\forall \phi_{1}\right)\left(\forall \phi_{2}\right)\left[\mathrm{I}\left(\phi_{1}\right)=\mathrm{I}\left(\phi_{2}\right)\right. \\
\leftrightarrow & \left((\forall z)(\forall w)\left(z \curvearrowleft\left(w \frown \phi_{1}\right) \rightarrow(\forall v)\left(z \curvearrowleft\left(v \frown \phi_{1}\right) \rightarrow w=v\right)\right)\right. \\
\leftrightarrow & \left.(\forall z)(\forall w)\left(z \curvearrowleft\left(w \frown \phi_{2}\right) \rightarrow(\forall v)\left(z \curvearrowleft\left(v \frown \phi_{2}\right) \rightarrow w=v\right)\right)\right]
\end{aligned}
$$

\footnotetext{
${ }^{23}$ We think it is worth noting that it seems likely to us that Frege himself started with this universally quantified second-order formula.

${ }^{24}$ We use Frege's application operator " $\cap$ " in order to capture Frege's official definition. We will say more about this operator below, for the moment it can be understood akin to membership.

${ }^{25}$ The remainder of Frege's Grundgesetze definitions, including definitions $\Delta, \mathrm{K}, \Lambda, \mathrm{N}, \Pi$ $\mathrm{P}, \Sigma, \mathrm{T}, \Upsilon, \Phi, \mathrm{X}, \Psi, \Omega, \mathrm{AA}, \mathrm{AB}, \mathrm{A} \Gamma$ follow a similar pattern. In future work we plan to show explicitly that all of these definitions result from straightforward application of the generalized recipe.
} 
In short, the truth-value denoted by $\mathrm{I} \phi_{1}$ is identical to the truth-value denoted by I $\phi_{2}$, if and only if the claim that $\phi_{1}$ is the double value-range of a single-valued relation (i.e. a function) is equivalent to the claim that $\phi_{2}$ is the double value-range of a single-valued relation. While this formula is complex, we can easily apply Step $\mathbf{2 . 5}$ by reminding ourselves that there is no distinction between logical equivalence and identity in Grundgesetze. Hence the right-hand-side of the above is equivalent to:

$$
\begin{aligned}
& \left((\forall z)(\forall w)\left(z \curvearrowleft\left(w \cap \phi_{1}\right) \rightarrow(\forall v)\left(z \cap\left(v \cap \phi_{1}\right) \rightarrow w=v\right)\right)\right. \\
= & \left.(\forall z)(\forall w)\left(z \curvearrowleft\left(w \frown \phi_{2}\right) \rightarrow(\forall v)\left(z \curvearrowleft\left(v \cap \phi_{2}\right) \rightarrow w=v\right)\right)\right]
\end{aligned}
$$

and we can now apply Step 3 to arrive at Frege's definition:

$$
\mathrm{I} x=(\forall z)(\forall w)(z \frown(w \cap x) \rightarrow(\forall v)(z \frown(v \frown x) \rightarrow w=v))
$$

We hope these examples suffice to show that Frege's Grundgesetze definitions share a certain structure which is characterised by the generalized recipe. What best explains the (surprising) uniformity of the Grundgesetze definitions is that Frege was aware of this recipe - or, at least, one that is very much like it - and so, we believe there are good grounds for thinking that Frege followed the generalized recipe when composing his magnum opus. ${ }^{26}$

Before concluding with a discussion of the consequences of this general definitional strategy for an adequate interpretation of Frege's mature philosophy of mathematics, there are three issues that need to be addressed: the first involves extant criticisms of Frege's definitions in Grundgesetze to the effect that his methodology is completely arbitrary. The second issue is to demonstrate that the generalized recipe can be applied in such a way as to avoid the problems that plagued the simple recipe - in particular, the problem of the singleton. The final issue is to examine closely the one exception to the generalized recipe, in order to show why this case must have been an exception on Frege's account.

\subsection{The Generalized Recipe and Arbitrariness}

Richard Heck (following Michael Dummett), has suggested that Frege's definitions in Grundgesetze are almost entirely arbitrary - that is, that Frege could have chosen just about any extensions whatsoever to be the referents of the various notions given explicit definitions in Grundgesetze:

\footnotetext{
${ }^{26}$ Admittedly, there is, as far as we know, no explicit mention of a recipe of this kind in Frege's published writing. According to (Veraart, 1976), Frege's Nachlaß contained many pages of "formulae" which could have offered us a better insight into how Frege arrived at the definition that he actually gives. As is well-known, however, most of the Nachlaß was lost during an air raid at the end of the second world war. See, however, (Wehmeier and Schmidt am Busch, 2005).
} 
In Frege: Philosophy of Mathematics, Michael Dummett argues that Frege's explicit definition of numerical terms is intended to serve just two purposes: To solve the Caesar problem, that is, to "fix the reference of each numerical term uniquely", and "to yield" HP ((Dummett, 1991), ch. 14). The explicit definition is in certain respects arbitrary, since numbers may be identified with a variety of different extensions (or sets, or possibly objects of still other sorts): there is, e.g., no particular reason that the number six must be identified with the extension of the concept "is a concept under which six objects fall"; it could be identified with the extension of the concept "is a concept under which only the numbers zero through five fall" or that of "is a concept under which no more than six objects fall".

(Heck, 2011), p. 95.

However, in a postscript added to this essay in the excellent collection entitled Frege's Theorem, Heck revises his earlier claims. He writes of the 'arbitrariness' charge:

This claim now seems to me to be over-stated, [...]. In particular, it now seems to me that there is a strong case to be made that the particular explicit definition that Frege gives - assuming that we are going to give an explicit definition - is almost completely forced. $[\ldots]$

So consider the matter quite generally. We have some equivalence relation $\xi R \eta$, and we want to define a function $\rho(\xi)$ in such a way as to validate the corresponding abstraction principle:

$$
\rho(x)=\rho(y) \leftrightarrow x R y
$$

How, in general, can we do this? So far as I can see, the only general strategy that is available here is essentially the one Frege adopts: Take $\rho(x)$ to be $x$ 's equivalence class under $R$, that is, the extension of the concept $x R \xi$.

(Heck, 2011), p. 109.

Heck would be absolutely right had Frege applied the simple recipe. In fact, the simple recipe, as we described it above, delivers exactly this result!

As we have already seen, however, the simple recipe is inadequate: it is susceptible to the problem of the singleton, and it does not generalize straightforwardly to non-unary abstracts. By the time of Grundgesetze, Frege had adopted the more powerful, but also more flexible, generalized recipe. As a result, the correct reading of Frege's mature Grundgesetze definitions is somewhere between the 'completely arbitrary' understanding 
suggested by the initial Heck quote and the 'completely forced' understanding suggested by the postscript. In fact, any of the definitions Heck considers in the passage above could be arrived at via the generalized recipe as 'the' definition of cardinal numbers.

Since constructions of such alternate definitions of cardinal numbers are familiar, we will illustrate this phenomenon with a different exampleFrege's definition of the ordered pair operation ";". Recall that we began our reconstruction of Frege's definition of ordered pairs (as, loosely speaking, sets of all relations that relate the objects in question in the appropriate order) by noting that the following provides the correct identity conditions.

$$
\forall \phi_{1}, \phi_{2}, \phi_{3}, \phi_{4} \in \Phi_{C}\left[\phi_{1} ; \phi_{2}=\phi_{3} ; \phi_{4} \leftrightarrow\left(\phi_{1}=\phi_{3} \wedge \phi_{2}=\phi_{4}\right)\right]
$$

Thus, Step 1 and Step 2 remain as before. The difference comes in how we carry out Step 2.5. Here, we will note that the right-hand side of the above is equivalent to:

$$
(\forall x)(\forall y)\left(\left(x=\phi_{1} \wedge y=\phi_{2}\right) \leftrightarrow\left(x=\phi_{3} \wedge y=\phi_{4}\right)\right)
$$

Two applications of Basic Law V then provide:

$$
\dot{\alpha} \dot{\varepsilon}\left(\varepsilon=\phi_{1} \wedge \alpha=\phi_{2}\right)=\dot{\alpha} \dot{\varepsilon}\left(\varepsilon=\phi_{3} \wedge \alpha=\phi_{4}\right)
$$

We now have the required identity, and can apply Step 3, resulting in the following definition of ordered pair:

$$
x ; y=\dot{\alpha} \dot{\varepsilon}(\varepsilon=x \wedge \alpha=y)
$$

In short, on this application of the generalized recipe, the ordered pair of $x$ and $y$ is not (speaking loosely) the set of all relations that holds of $x$ and $y$ (in that order), but is instead the single relation that relates $x$ to $y$ (again, in that order) and relates nothing else to anything else. ${ }^{27}$

Thus, the generalized recipe does not generate a unique definition, but it is instead a general method for arriving at one of a number of equally adequate definitions. As a result, there is a measure of arbitrariness present in Frege's mature account of definitions in Grundgesetze. This point should not be overstated, however: The method does not license an 'anything-goes' approach to definition. In particular, it is not the case that given any acceptable definition of the form:

$$
f\left(x_{1}, x_{2}, \ldots x_{n}\right)=\Phi\left(x_{1}, x_{2}, \ldots x_{n}\right)
$$

\footnotetext{
${ }^{27}$ Other paths to the requisite identity on the right hand side are possible. For example, one can carry out Step $\mathbf{2 . 5}$ in such a way as to arrive at a Fregean version of the Kuratowski definition of ordered pair - that is:

$$
x ; y=\dot{\varepsilon}(\varepsilon=\dot{\alpha}(\alpha=x) \vee \varepsilon=\dot{\alpha}(\alpha=x \vee \alpha=y))
$$

Details are left to the reader.
} 
and any arbitrary one-to-one function $g$, that:

$$
f\left(x_{1}, x_{2}, \ldots x_{n}\right)=g\left(\Phi\left(x_{1}, x_{2}, \ldots x_{n}\right)\right)
$$

is also an acceptable definition. On the contrary, according to the generalized recipe, any acceptable definition must proceed by moving from appropriate identity conditions (Step 2) via logical laws (including Basic Law V) to an appropriate identity (Step 2.5). Thus, while the generalized recipe is open-ended - sanctioning more than one possible definition but, presumably, allowing no more than one at once - it does not sanction just any definition that might get the identity conditions correct.

A final question remains: Why did Frege select the particular definitions that he did select, rather than one or another of the other possibilities? Here we can at best speculate, but we suspect the answer will lie in a combination of two factors. First, there is the issue of technical convenience. Some generalized recipe definitions of a particular concept will be more fruitful or more economical than others in terms of the role they play in the constructions and proofs that Frege wishes to carry out in Grundgesetze. Second, there is the issue of applications and what is now called Frege's Constraint - the thought that an account of the application of a mathematical concept should flow immediately from the definition of that concept (see, e.g. (Frege, 1903), §159, p. 157). Clearly, some definitions will satisfy Frege's Constraint more easily and more straightforwardly than others. ${ }^{28}$

Making such judgements with regard to one proposed definition rather than another will not always be simple, however. At an intuitive level, both the convenience/fruitfulness/economy consideration and the Frege's Constraint consideration seem to weigh in favor of Frege's preferred definition of number rather than any of the alternative constructions suggested by Heck. But the case for Frege's preferred definition of ordered pair, rather than the alternative construction just given, is not so clear. It will require a detailed examination of the role that ordered pairs play in the derivations of Grundgesetze and the way the notion of pair is applied more generally. For now, since we have other fish to fry, we will remain content having raised this interpretational question. ${ }^{29}$

\subsection{Arbitrariness and the problem of the singleton}

Since we began this section with the observation that the explicit definitions given in Grundlagen can be recaptured by application of the generalized recipe, the natural question to ask next is whether an application of the

\footnotetext{
${ }^{28}$ An obvious third consideration is simplicity. Thus, it would be perverse for Frege to identify numbers with the singletons of the objects that he does identify as numbers, even though such a definition can be obtained via the generalized recipe and does get the identity conditions for numbers correct.

${ }^{29}$ Needless to say, we plan to return to this issue in future work.
} 
generalized recipe to extensions themselves will fall prey to the problem of the singleton. The answer to this question is, in an interesting and important sense, "yes" and "no". In more detail: some applications of the generalized recipe do run afoul of the problem of the singleton, but not all do.

In applying the generalized recipe to extensions, Step $\mathbf{1}$ and Step $\mathbf{2}$ are straightforward: the concept $C$ in question is ExTENSION, the underlying concept $\Phi_{C}$ such that $C$ 's are $C$ 's of $\Phi_{C}$ 's is the concept (FIRST-LEVEL) CONCEPT, and the appropriate equivalence relation on concepts is coextensionality. Thus, we have:

$$
\dot{\varepsilon}(F(\varepsilon))=\dot{\varepsilon}(G(\varepsilon)) \leftrightarrow(\forall x)(F(x) \leftrightarrow G(x))
$$

We can now apply Basic Law V to the right-hand side, and obtain:

$$
\dot{\varepsilon}(F(\varepsilon))=\dot{\varepsilon}(G(\varepsilon)) \leftrightarrow \dot{\varepsilon}(F(\varepsilon))=\dot{\varepsilon}(G(\varepsilon))
$$

With Step 2.5 completed, we can apply Step 3 and obtain the following innocuous identity:

$$
\dot{\varepsilon}(F(\varepsilon))=\dot{\varepsilon}(F(\varepsilon))
$$

So far, so good - the most natural way of applying the generalized recipe turns out to be immune to the problem of the singleton.

The problem is that Step 2.5 - the real culprit in the arbitrariness issue - only requires that we transform the right-hand side of the abstraction principle formulated in Step $\mathbf{2}$ into an identity. It does not provide any particular guidance on how to do so, nor does it guarantee that there will be only one such identity that can be reached via the application of basic laws and rules of inference. Thus, in carrying out Step 2 above, we could have applied Basic Law V to the right-hand side to obtain:

$$
\dot{\varepsilon}(F(\varepsilon))=\dot{\varepsilon}(G(\varepsilon)) \leftrightarrow \dot{\varepsilon}(F(\varepsilon))=\dot{\varepsilon}(G(\varepsilon))
$$

then applied some basic logic to obtain:

$$
\dot{\varepsilon}(F(\varepsilon))=\dot{\varepsilon}(G(\varepsilon)) \leftrightarrow(\forall x)(x=\dot{\varepsilon}(F(\varepsilon)) \leftrightarrow x=\dot{\varepsilon}(G(\varepsilon)))
$$

and then applied Basic Law V again to obtain:

$$
\dot{\varepsilon}(F(\varepsilon))=\dot{\varepsilon}(G(\varepsilon)) \leftrightarrow \dot{\alpha}(\alpha=\dot{\varepsilon}(F(\varepsilon)))=\dot{\alpha}(\alpha=\dot{\varepsilon}(G(\varepsilon)))
$$

Applying Step 3 at this stage would result in the following identification:

$$
\dot{\varepsilon}(F(\varepsilon))=\dot{\alpha}(\alpha=\dot{\varepsilon}(F(\varepsilon)))
$$

This, however, is exactly the identity that got us into trouble in the first place. Thus, the generalized recipe can be applied safely to extensions themselves, but not all such applications are safe. What, then, does this tell us about 
the arbitrariness of the generalized recipe itself, and how we are meant to apply it in potentially problematic cases?

One possible response is to formulate some additional principles guiding the application of the recipe - principles that legitimate the first of the two applications of the generalized recipe to extensions, while ruling out the second application as illegitimate. Supplementing the recipe in this manner, if it were possible, could perhaps be done in such a way as to eliminate all arbitrariness whatsoever, salvaging the idea that Frege's methods provide a unique definition of each mathematical concept. Such an account would be attractive, but let us raise a problem for it (though there might be many more).

It is not clear how to formulate such constraints on Step 2.5 in the first place. In comparing the two constructions above, one is immediately struck by the fact that, in the second, problematic construction, we had already obtained an identity of the requisite form:

$$
\dot{\varepsilon}(F(\varepsilon))=\dot{\varepsilon}(G(\varepsilon)) \leftrightarrow \dot{\varepsilon}(F(\varepsilon))=\dot{\varepsilon}(G(\varepsilon))
$$

but then continued to manipulate the right-hand side until we had obtained a second such identity, to which an application of Step $\mathbf{3}$ provided the problem of the singleton-susceptible definition. Thus, one natural thought is to require that the application of Step $\mathbf{2 . 5}$ terminate at the first instance of an appropriate identity on the right-hand side. While such a rule would block the second construction above, it does not block an alternate construction that terminates with the same identity, and hence (via application of Step 3) provides the same problematic identification of extensions with their singletons. We begin with the same equivalence relation on the right, and, applying some straightforward logic, arrive at:

$$
\dot{\varepsilon}(F(\varepsilon))=\dot{\varepsilon}(G(\varepsilon)) \leftrightarrow(\forall H)((\forall x)(H(x) \leftrightarrow F(x)) \leftrightarrow(\forall x)(H(x) \leftrightarrow G(x)))
$$

Two applications of Basic Law V provide us with:

$$
\dot{\varepsilon}(F(\varepsilon))=\dot{\varepsilon}(G(\varepsilon)) \leftrightarrow(\forall H)(\dot{\varepsilon}(H(\varepsilon))=\dot{\varepsilon}(F(\varepsilon)) \leftrightarrow \dot{\varepsilon}(H(\varepsilon))=\dot{\varepsilon}(G(\varepsilon)))
$$

We then obtain:

$$
\dot{\varepsilon}(F(\varepsilon))=\dot{\varepsilon}(G(\varepsilon)) \leftrightarrow(\forall x)(x=\dot{\varepsilon}(F(\varepsilon)) \leftrightarrow x=\dot{\varepsilon}(G(\varepsilon)))
$$

via more logic, and apply Basic Law $\mathrm{V}$ in order to obtain the problematic identity:

$$
\dot{\varepsilon}(F(\varepsilon))=\dot{\varepsilon}(G(\varepsilon)) \leftrightarrow \dot{\alpha}(\alpha=\dot{\varepsilon}(F(\varepsilon)))=\dot{\alpha}(\alpha=\dot{\varepsilon}(G(\varepsilon)))
$$

Thus, requiring that Step $\mathbf{2 . 5}$ halts at the first appropriate identity does not block the problematic construction. ${ }^{30}$

\footnotetext{
${ }^{30}$ Note that, although Frege does not distinguish between biconditionals and identities, the intermediate formulas in the construction above involve universal quantifications of identities/biconditionals, and hence are not identities themselves.
} 
That being said, there obviously is something fishy about the implementations of the generalized recipe that results in the problem of the singleton. Of course, it is possible that Frege would have rejected these constructions based on the sort of consideration discussed in the previous section: they introduce an understanding of the concept EXTENSION that is less convenient, less fruitful, and less simple than the original construction (and maybe they also violate Frege's Constraint). But there is another reason for rejecting them as legitimate applications of the generalized recipe: they violate logical constraints on the provision of adequate identity conditions for mathematical objects. Since the generalized recipe proceeds via explicit consideration of such identity conditions, it seems plausible that any application of the recipe should, in the end, respect such constraints. Frege was well aware of the need to respect logical and metaphysical constraints when proposing identities: Frege's permutation argument in $\S 10$ of Grundgesetze is, in effect, an argument which shows that identifying the truth values with their singletons will not generate logical difficulties of exactly the sort that would arise were he to identify all objects with their singletons more generally. ${ }^{31}$

This provides an additional criterion by which Frege might judge particular applications of the recipe, and which can thus be used to help explain why he arrived at the particular definitions codified in Grundgesetze: in addition to respecting considerations of simplicity and fruitfulness, and adhering to Frege's Constraint, applications of the generalized recipe should not bring with them logical difficulties of the sort exemplified by the problem of the singleton. ${ }^{32}$ From this perspective, then, the fact that there is a well-motivated implementation of the generalized recipe that does not give rise to the problem of the singleton, might well be enough to regard the generalized recipe to be in good standing with respect to that very problem.

\footnotetext{
${ }^{31}$ Of course, the identification of truth values with their singletons is, as we have already emphasized, not carried out via an official definition or axiom within the formal system of Grundgesetze, but is instead merely a 'meta'-level methodological stipulation. Nevertheless, the discussion in $\S 10$ of Grundgesetze makes it clear that Frege was explicitly aware of the sort of logical constraints that weigh in favor of the simpler application of the generalized recipe to extensions.

${ }^{32}$ Note that the sort of logical difficulty at issue is not restricted to applications of the generalized recipe to extensions, but would also apply if Frege were to codify his identification of truth values with their singletons within the formal system of Grundgesetze. Similar logical constraints would govern cases where the generalized recipe were applied to two distinct concepts with non-disjoint extensions, since the definitions would need to be logically compatible on those objects falling under both concepts.
} 


\subsection{The exception to the generalized recipe}

The only exception to the generalized recipe is definition A-the definition of the application operation " $\cap$ ":

$$
\Vdash \backslash \dot{\alpha}\left(\sim^{\mathfrak{g}}\left[\begin{array}{c}
\mathfrak{g}(a)=\alpha \\
u=\dot{\varepsilon} \mathfrak{g}(\varepsilon)
\end{array}\right)=a \wedge u\right.
$$

$a^{\wedge} u$ is the value of the function $f$ applied to the argument $a$ where $u$ is the value-range of $f$ (when $u$ is not a value-range, then $a \cap u$ refers to the value-range of the function that maps every object to the false - that is, to $\dot{\varepsilon}\left({ }_{\mathrm{T}} \varepsilon=\varepsilon\right)$.)

Of particular interest is the case where $u$ is the extension of a concept $C$ (that is, $C$ is a function from objects to truth-values), where $a \cap u$ will be the True if $C$ holds of $a$, and the False otherwise. As a result, when applied to extensions, $\cap$ is, in effect, a Fregean analogue of the set-theoretic membership relation $\in$, and Frege often uses $\wedge$ as a membership relation on extension of concepts. ${ }^{33}$

What is most notable about $\cap$ for our purposes, however, is that it is an exception to the account of the Grundgesetze definitions sketched above: Freges application operator $\cap$ is neither a definition of a specific object nor is it the result of an application of the generalized recipe to obtain definitions of unary predicates or definitions of binary functions on value-ranges, but it is a unique fourth case. It is therefore likely no accident that this is the very first definition Frege provides in Grundgesetze, since it not only plays a critical role in the later constructions (as a quick perusal of its use in the remaining definitions and central theorems makes clear), but it also plays a unique role in Frege's approach to definition.

In order to see why definition A is special, it is worth working through what would result if we attempted to arrive at a definition of " $\cap$ " via the generalized recipe. $\cap$ is a function that takes two objects as arguments, and, when the latter argument is the value-range of a function, gives the value of that function applied to the first argument. Hence, applying Step $\mathbf{1}$ and Step 2 of the recipe, we obtain something like:

$$
\begin{aligned}
& (\forall x)(\forall y)(\forall z)(\forall w)[x \cap y=z \cap w \\
\leftrightarrow & (\forall v)[(\exists f)(y=\dot{\varepsilon}(f(\varepsilon) \wedge f(x)=v) \\
\leftrightarrow & (\exists f)(w=\dot{\varepsilon}(f(\varepsilon) \wedge f(z)=v)]
\end{aligned}
$$

Via Basic Law V, we can see that the right-hand side of the formula above is equivalent to:

$$
\dot{\varepsilon}((\exists f)(y=\dot{\varepsilon}(f(\varepsilon) \wedge f(x)=\varepsilon))=\dot{\varepsilon}((\exists f)(w=\dot{\varepsilon}(f(\varepsilon) \wedge f(z)=\varepsilon))
$$

\footnotetext{
${ }^{33}$ Frege himself glosses this operation as the "Relation of an object falling within the extension of a concept" (Frege, 1893), p. 240.
} 
With the identity required by Step $\mathbf{2 . 5}$ in hand, we can then suggest the following definition:

$$
x \cap y=\dot{\varepsilon}((\exists f)(y=\dot{\varepsilon}(f(\varepsilon) \wedge f(x)=\varepsilon))
$$

This definition gets the identity conditions right, but there is an immediate, and obvious, problem: This definition does not give us the value of the function $f$ applied to argument $x$, where $y=\dot{\varepsilon}(f(\varepsilon))$, but instead provides us with the singleton of $f(x)$. As we have already shown, however, Frege was quite aware of the dangers of haphazardly conflating objects with their singletons, so it should come as no surprise that Frege does not adopt the incorrect definition above, but instead applies the 'singleton-stripping' 34 operation \to this formulation, obtaining the correct definition:

$$
x \cap y=\backslash \dot{\varepsilon}((\exists f)(y=\dot{\varepsilon}(f(\varepsilon) \wedge f(x)=\varepsilon))
$$

Thus, Definition A is the sole exception to the generalized recipe since it requires an additional step.

Why is Definition A different from the remaining definitions in Grundgesetze? The answer is surprisingly straightforward. Throughout the rest of Grundgesetze, each definition introduces a new concept, function, or other operation by identifying the range of that concept, function, or operation with a sub-collection of the universe of value-ranges. In short, Frege is defining new concepts by identifying their ranges with objects taken from the old, and constant, domain. As a result, it is sufficient for his purposes in these cases merely to identify some objects with the right identity conditions (modulo the possible additional constraints touched on in the previous subsections), and this is exactly what the generalized recipe accomplishes.

With the definition of $\neg$ something very different is going on. In this case, Frege is not attempting to introduce some new concept, instead he is attempting to formulate a new way of getting at an already understood and fully specified operation-function application. As Frege puts it:

It has already been observed in $\S 25$ that first-level functions can be used instead of second-level functions in what follows. This will now be shown. As was indicated, this is made possible by the fact that the functions appearing as arguments of secondlevel functions are represented by their value-ranges, although of

\footnotetext{
${ }^{34}$ The 'singleton stripping' (or backslash) operator is a unary function from objects to objects such that (compare (Frege, 1893), §11, p. 19):

$$
\begin{aligned}
f(a) & =b & \text { if } a=\dot{\varepsilon}(b=\varepsilon) \\
& =a & \text { otherwise. }
\end{aligned}
$$

In short, Frege's backslash is an object-level function that, when applied to the extension of a concept, serves the same purpose as a Rusellian definite description operator when applied directly to that concept.
} 
course not in such a way that they simply concede their places to them, for that is impossible. In the first instance, our concern is only to designate the value of the function $\Phi(\xi)$ for the argument $\Delta$, that is, $\Phi(\Delta)$, using ' $\Delta$ ' and ' $\dot{\varepsilon} \Phi(\varepsilon)$ '.

(Frege, 1893), §34, p. 52 .

In short, Frege needs a definition of " $\cap$ " that not only guarantees that the objects 'introduced' have the right identity conditions, but in addition that they are the right objects. As a result, Definition A is of a very different sort than the definitions that follow it, and so it should not be surprising that it does not follow the pattern provided by the generalized recipe.

\section{Applications and consequences}

We believe that the general recipe not only provides an accurate and illuminating rational reconstruction of Frege's method of definition in Grundgesetze, but that he knowingly applied this methodology (or something very similar). As mentioned before, it would be hard to explain the uniformity of the Grundgesetze definitions if Frege did not have a methodological template of this sort in mind. However, we shall not here offer a further defence of the claim that Frege's use of the generalized recipe was explicit. Instead, we shall conclude by showing how awareness and appreciation of the role of the generalized recipe in Frege's Grundgesetze can shed a new light on a number of difficult interpretative issues in Frege scholarship. ${ }^{35}$

\subsection{The role of Basic Law V in Grundgesetze}

As has been shown by Richard Heck ${ }^{36}$, Frege did not make much real use of Basic Law V in the derivations found in part II of Grundgesetze-Frege's only ineliminable appeal to Basic Law $\mathrm{V}$ is in deriving each direction of Hume's Principle. Most other occurrences of value-ranges, and applications of Basic Law V to manipulate them, are easily eliminable. This raises a fundamental question about the role of Basic Law V in Frege's philosophy of mathematics - one forcefully formulated by Heck:

How can an axiom which plays such a limited formal role be of such fundamental importance to Frege's philosophy of mathematics?

(Heck, 2011), p. 65.

\footnotetext{
${ }^{35}$ Here we will address only two such topics, but we believe that the account of definition given here can also provide insights into the Caesar problem, Frege's reconstruction of real analysis, and his views on geometry, amongst other things. We plan on returning to these topics in future work.

${ }^{36}$ See his (Heck, 1993).
} 
Clearly, Frege did attach fundamental importance to Basic Law V. Consider, for example, the Afterword of Grundgesetze, where, faced with Russell's paradox, he attempts to provide a 'correction' to his conception of valueranges. Frege does not, as might be expected given the limited formal role that Basic Law V plays, suggest that we abandon extensions altogether, but instead suggests that a slight modification of our understanding of value-ranges is all that is needed:

So presumably nothing remains but to recognise extensions of concepts or classes as objects in the full and proper sense of the word, but to concede at the same time that the erstwhile understanding of the words "extension of a concept" requires correction.

(Frege, 1903), Afterword, p. 255-56 (our italics).

After he introduces the principle that introduces the 'improved' understanding of extensions - Basic Law $V^{\prime}$ - he closes the Afterword by stating that:

This question may be viewed as the fundamental problem of arithmetic: how are we to apprehend logical objects, in particular, the numbers? What justifies us to acknowledge numbers as objects? Even if this problem is not solved to the extent that I thought it was when composing this volume, I do not doubt that the path to the solution is found.

(Frege, 1903), Afterword, p. 265.

So, for Frege there is no doubt that something in the spirit of Basic Law V captures the "characteristic constitution" of value-ranges, and that valueranges play a central role in his philosophical project - a role they continue to play even when confronted with the paradox. But how are we to square Frege's insistence on the importance of Basic Law V (or some variant of it such as Basic Law $\mathrm{V}^{\prime}$ ) with the limited formal role that it plays in the derivations of Grundgesetze?

Our interpretation of Frege's Grundgesetze highlights a central role played by Basic Law $\mathrm{V}$ - one distinct from its role as an axiom within the formal system of Grundgesetze. The generalized recipe relies fundamentally on Basic Law V (or, more carefully, on a metatheoretic analogue of Basic Law V which is first introduced in vol. I, $\S 3$ and $\S 9$ ), since applications of Basic Law V are required (in most cases) in order to move from the statement of identity conditions (Step 2) to the required identity between objects (Step 2.5). Thus, the role played by Basic Law V (and, later, by Basic Law $V^{\prime}$ ) is broader than merely providing the identity conditions for value-ranges! Instead, it also plays a central role in identifying which value-ranges are the objects 'falling under' all other mathematical concepts. In short, its role is not only logical - as a central principle of the formal system of Grundgesetze-but 
also epistemological and metaphysical, since it is a central component of the method by which we define mathematical concepts and identify mathematical objects such as cardinal numbers and ordered pairs. As a result, and in retrospect, it should not be too surprising that Basic Law V plays a limited role in the formal proofs of Grundgesetze, since this formal work consists merely of unpacking the real work carried out by Basic Law V: the (informal, metatheoretical) formulation of accurate and adequate definitions prior to formal derivations - that is, its role in the generalized recipe. ${ }^{37}$

\subsection{The role of Hume's Principle in Grundgesetze}

A second issue of interest here, and extensively discussed in Richard Heck's writings $^{38}$, is the role of Hume's Principle in Frege's mature philosophy of mathematics. As we noted in section 1, Frege appeals to Hume's Principle in $\S 62$ of Grundlagen when attempting to explain how numbers are given to us. Frege ultimately rejects Hume's Principle as a definition of number and opts instead for the explicit definition of cardinal numbers as a type of extension. Nevertheless, Frege explicitly requires that any value-range based definition should allow us to prove Hume's Principle, and Hume's Principle continues to plays a central role throughout the remainder of Grundlagen.

Given the continued appearance of Hume's Principle (and similar informal principles) throughout Grundlagen, we think that this principle played two separate (but interrelated) roles in Frege's philosophy of mathematics at this point even after it was rejected as a definition. First, Hume's Principle as an informal meta-theoretical principle provides the correct identity conditions for cardinal numbers and guides the formulation of a definition of cardinal numbers (i.e. whatever extensions are chosen, they must have the identity conditions codified by Hume's Principle). Second, Hume's Principle, as a formula of the - in Grundlagen informal - object language, constitutes an adequacy condition on any explicit definition of cardinal numbers in terms of extensions (or in terms of anything else, for that matter): whatever definition we choose, it must demonstrably provide the right identity conditions; the way to provide such a guarantee is to require that it proof-theoretically entails the formula that codifies those identity conditions - that is, the definition must entail Hume's Principle.

This all seems straightforward enough, but we now arrive at a puzzle: why is it that Frege does not mention Hume's Principle, or even explicitly prove it in full biconditional form, in Grundgesetze? Frege does prove each direction individually, but he does not put them together into a biconditional/identity claim. As already noted, Frege does not explicitly prove Hume's Principle in full in Grundlagen either, but the proof sketch of the right-to-left direction

\footnotetext{
${ }^{37}$ In (Ebert and Rossberg, 2016b) we argue that Frege draws on exactly this further role of BLV in the rather intriguing passages $\S 146$ and $\S 147$ of volume II of Grundgesetze.

${ }^{38}$ See for example (Heck, 1995) and (Heck, 2005).
} 
in $\S 73$, plus the footnote at the end of the same section addressing the left-toright direction, are, we think, meant to jointly indicate the existence of such a proof. Moreover, Frege often talks of Hume's Principle in biconditional form in the prose in Grundlagen. In contrast, in Grundgesetze the two directions of Hume's Principle are proven in different chapters ( $A$ and $B$ respectively) with no indication that they are to be 'put together' or that anything might be gained by doing so. ${ }^{39}$ Also, there is no mention of Hume's Principle as a biconditional in the prose of Grundgesetze. Interestingely, the sections of Grundgesetze where the definition of natural number is provided (§§38-46) refer to $\S 68$ of Grundlagen (where the explicit definition of cardinal number is first given), $\S \S 71-72$ of Grundlagen (where the definition of equinumerosity is formulated), and $\S \S 74-79$ of Grundlagen (where explicitly definitions of 0,1 , and successor are formulated, and sketches of the Peano axioms are given). Striking in its absence is any mention of $\S 73$ of Grundlagen where the sketch of the proof of Hume's Principle is given. ${ }^{40}$ Taken together, this suggests that the role of abstraction principles in Grundgesetze has changed and that Hume's Principle, understood as a constraint on any adequate definition of cardinal number, has disappeared in Grundgesetze. How are we to reconcile the fundamentality of Hume's Principle in the philosophy of the Grundlagen-Frege with the fact that it plays a far less important role in the philosophy of the Grundgesetze-Frege?

Once we are aware of the difference between the simple recipe and the generalized recipe, an explanation is not hard to come by. Sometime between Grundlagen and Grundgesetze Frege must have realized that, if Step 2 of the generalized recipe is carried out correctly - that is, if, in the case of cardinal numbers, Hume's Principle (or the metatheoretical analogue given above) is used to provide the identity conditions for cardinal numbers - then Step 4 of the simple recipe is redundant. There simply is no need to proof-theoretically establish Hume's Principle, qua abstraction principle, within the formalism of Grundgesetze so long as the generalized recipe is carried out correctly, and nothing that is of philosophical or mathematical importance would be

\footnotetext{
${ }^{39}$ We owe this important observation to (May and Wehmeier, 2016). Although they give a different explanation for this odd fact than the one given here. They are the first to suggest that Frege's failure to 'conjoin' the two directions of Hume's Principle is not merely a technical quirk of the organization of Grundgesetze, but instead provides insights into what Frege was up to. Thus, our own discussion owes much to their careful examination of these issues.

${ }^{40}$ Frege does indeed mention $\S 73$ of Grundlagen later, in a footnote which we reproduce in its entirety:

Compare Grundlagen, p. 86.

(Frege, 1893) §54, p. 72 (fn1).

This footnote does not concern the derivation of Hume's Principle in Grundlagen $\S 73$, however, but merely highlights the fact that Frege's definition and elucidation of the composition relation in Grundgesetze $\S 56$ is based on notions first presented in a subportion of that derivation.
} 
achieved by putting together both sides of Hume's Principle and proving the formal counterpart in the language of Grundgesetze.

As a final observation, it is worth noting that these points might also help to explain why Frege was not at all tempted to use Hume's Principle as a definition of cardinal number after he became aware of Russell's paradox, especially given Frege was arguably aware of the fact that Hume's Principle alone would entail all of the Peano axioms. ${ }^{41}$ Dropping Basic Law V leaves Frege without a general means for defining mathematical objects - that is, it forces him to abandon the generalized recipe (and the simple recipe, for that matter) altogether. Hume's Principle, or its metatheoretical analogue, can (and does) provide the right identity conditions for cardinal numbers, but it is insufficient to pick out which objects the cardinal numbers are. Hume's Principle simply cannot play the epistemological and metaphysical role that Basic Law V was meant to play in Grundgesetze. Thus, without Basic Law V (or some variant, such as Basic Law V') Frege was left with no means for defining and thereby introducing mathematical objects, and hence no identifiable mathematical objects at all. This observation is, of course, in stark contrast to the recent neo-logicist attempt to found arithmetic on Hume's Principle. In the following, we want to highlight one more important difference between the two approaches. ${ }^{42}$

\subsection{The definitional strategy and neo-logicism}

Finally, it is worth observing that the ontology presupposed and utilized by Frege in his application of the generalized recipe - or even the simple recipe-differs markedly from recent neo-logicist approaches as defended in (Wright, 1983), (Hale and Wright, 2001). ${ }^{43}$ Despite their Fregean roots, neo-logicists reject the idea that objects falling under some mathematical concept $C$ should be identified with corresponding extensions or value-ranges. Instead, given a mathematical concept $C$, the neo-logicist will provide an abstraction principle of the form:

$$
(\forall \alpha)(\forall \beta)\left[@_{C}(\alpha)=@_{C}(\beta) \leftrightarrow E_{C}(\alpha, \beta)\right]
$$

that defines the concept $C$ by providing identity conditions (via the equivalence relation $E_{C}(\ldots, \ldots)$ ) for abstract objects falling under $C$ (the referents of abstraction terms $\left.@_{C}(\ldots)\right)$. Hence, on the neo-logicist approach cardinal numbers and other abstract objects are not identified as being amongst some more inclusive, previously identified range of objects. As result, a neo-logicist

\footnotetext{
${ }^{41}$ In fact, he does briefly consider, and immediately rejects, this option in a letter to Russell. Compare (Gabriel et al., 1976), XXXVI/7, p. 224.

${ }^{42}$ Compare (Blanchette, 2016), who highlights further differences between the "Scottish" neo-logicist and Frege's logicism.

${ }^{43}$ For an overview of issues concerning this form of neo-logicism, see the introduction to (Ebert and Rossberg, 2016a).
} 
does not require anything akin to Frege's recipe and, thus, she is not plagued by the sort of limited arbitrariness discussed previously: the abstract objects falling under mathematical concepts just are whatever objects are delineated by (acceptable) abstraction principles.

This plenitude of kinds of abstract objects comes at a cost, however: the neo-logicist owes us a principled account of the truth-conditions of cross-abstraction identity statements of the form:

$$
@_{C_{1}}(\alpha)=@_{C_{2}}(\beta)
$$

where $C_{1}$ and $C_{2}$ are different mathematical concepts, defined by different abstraction principles. This problem has come to be called the $\mathbb{C}-\mathbb{R}$ problem. ${ }^{44}$

In contrast, such cross-abstraction identities are easily resolved by Frege: given two mathematical objects whose identity or distinctness might be in question, we need merely determine which extensions the generalized recipe identifies with those objects, and then apply Basic Law V to settle the identity claim in question. ${ }^{45}$ Of course, given the arbitrariness in the generalized recipe, it is possible that two objects that have been defined in such a way as to be distinct might have been defined in some other manner such that they would have been identical. But once a particular choice is made, there is no $\mathbb{C}-\mathbb{R}$ problem within Frege's original logicist project as developed in Grundgesetze.

As a result, we can now understand one aspect of the relation between Frege's logicism and his modern day neo-logicist successor in terms of adopting different approaches to a particular trade-off: Frege, in adopting the generalized recipe, was forced to accept some arbitrariness with regard to how he defined mathematical concepts such as CARDINAL NUMBER and ORDERED PAIR. Once he has settled on particular definitions, however, there are no further questions regarding identity claims holding between mathematical objects: all such objects are extensions (or value-ranges more generally) and so Basic Law $\mathrm{V}$ will settle the relevant identity in question. The neo-logicist, on the other hand, in rejecting the recipe - and a single domain of primitive objects generally - in favor of a multitude of distinct abstraction principles describing distinct (yet possibly overlapping) domains of mathematical objects,

\footnotetext{
${ }^{44}$ The name is a play on the familiar phrase "the Caesar problem", and refers to the specific case of determining whether the real numbers $\mathbb{R}$ generated by one abstraction principle are identical to a sub-collection of the complex numbers $\mathbb{C}$ given by a distinct abstraction principle. For a fuller discussion of this problem, see (Cook and Ebert, 2005) and more recently (Mancosu, 2015).

${ }^{45}$ We do not mean to imply that settling whether two extensions in a non-well-founded theory of extensions such as that found within Grundgesetze (or consistent sub fragments of Grundgesetze is trivial, effective, etc. The point is merely that on Frege's view the recipe entails that there will be a straightforward fact of the matter that settles these identities. Hence, regardless of whether determination of the status of cross-abstraction identities is in-principle mathematically difficult, there are no deep philosophical puzzles here.
} 
suffers from no such arbitrariness. But the cost of avoiding the arbitrariness found in Frege's project is the $\mathbb{C}-\mathbb{R}$ problem. ${ }^{46}$

\section{References}

Blanchette, P. (2012). Frege's Conception of Logic. Oxford University Press.

Blanchette, P. (2016). The breadth of the paradox. Philosophia Mathematica, 24(1):30-49.

Boolos, G. (1989). Iteration again. Philosophical Topics, 42:5-21. Reprinted in (Boolos, 1998).

Boolos, G. (1990). The standard of equality of numbers. In Boolos, G., editor, Meaning and Method: essays im honor of Hilary Putnam. Cambridge University Press, Cambridge. Reprinted in (Boolos, 1998).

Boolos, G. (1998). Logic, Logic and Logic. Harvard, Cambridge, Massachusetts.

Boolos, G. and Heck, Jr., R. G. (1998). Die Grundlagen der Arithmetik $\S \S 82-83$. In (Schirn, 1998), pages 407-428. Reprinted in (Boolos, 1998), pages $315-338$.

Cook, R. T. (2014). Frege's conception of logic, Patrica A. Blanchette. Philosophia Mathematica, 22(1):108-120.

Cook, R. T. (2015). Comments on Patricia Blanchette's book: Frege's conception of logic. Journal for the History of Analytical Philosophy, $3(7)$.

Cook, R. T. and Ebert, P. A. (2005). Abstraction and identity. Dialectica, $59: 121-139$.

Dummett, M. (1991). Frege: Philosophy of Mathematics. Harvard University Press, Cambridge, MA.

Ebert, P. A. and Rossberg, M., editors (2016a). Abstractionism. Oxford University Press, Oxford.

\footnotetext{
${ }^{46}$ We would like to thank the audience of an Early Analytic Group meeting at the University of Stirling and the audience of the MidWestPhilMathWorkshop 13 for valuable feedback. Furthermore, we would like to thank two anonymous referees for their extensive and challenging comments which led numerous improvements. This work was supported by a grant awarded to Philip Ebert from the UK Arts and Humanities Research Council: $\mathrm{AH} / \mathrm{J} 00233 \mathrm{X} / 1$.
} 
Ebert, P. A. and Rossberg, M. (2016b). Mathematical creationism in Frege's Grundgesetze? In Ebert, P. A. and Rossberg, M., editors, Essays on Frege's Basic Laws of Arithmetic. Oxford University Press.

Frege, G. (1884). Die Grundlagen der Arithmetik. Eine logisch mathematische Untersuchung über den Begriff der Zahl. Wilhelm Koebner, Breslau. English translation of the quoted passages were provided by the author.

Frege, G. (1893). Grundgesetze der Arithmetik. Begriffsschriftlich abgeleitet.

I. Band. Verlag H. Pohle, Jena. English translation in (Frege, 2013).

Frege, G. (1903). Grundgesetze der Arithmetik. Begriffsschriftlich abgeleitet. II. Band. Verlag H. Pohle, Jena. English translation in (Frege, 2013).

Frege, G. (2013). Basic Laws of Arithmetic. Oxford University Press, Oxford. Translated by Philip A. Ebert and Marcus Rossberg.

Gabriel, G., Hermes, H., Kambartel, F., Thiel, C., and Veraart, A., editors (1976). Gottlob Frege: Wissenschaftlicher Briefwechsel. Meiner, Hamburg. Partial English Translation: (Gabriel et al., 1980).

Gabriel, G., Hermes, H., Kambartel, F., Thiel, C., Veraart, A., and McGuinness, B., editors (1980). Gottlob Frege: Philosophical and Mathematical Correspondence. Translated by H. Kaal. University of Chicago Press, Chicago.

Hale, B. and Wright, C. (2001). The Reason's Proper Study: Essays towards a Neo-Fregean Philosophy of Mathematics. Clarendon Press.

Heck, Jr, R. G. (1993). The Development of Arithmetic in Frege's Grundgesetze der Arithmetik. Journal of Symbolic Logic, 58:579-601. Reprinted in (Heck, 2011).

Heck, Jr, R. G. (1995). Frege's principle. In Hintikka, J., editor, From Dedekind to Gödel: Essays on the Development of the Foundations of Mathematics, pages 119-45. Kluwer Academic Publishers, Dordrecht. Reprinted in (Heck, 2011).

Heck, Jr, R. G. (2005). Julius Caesar and Basic Law V. Dialectica, 59(161-78). Reprinted in (Heck, 2011).

Heck, Jr, R. G. (2011). Frege's Theorem. Oxford University Press.

Mancosu, P. (2015). In Good Company? On Hume's Principle and the assignment of numbers to infinite concepts. Review of Symbolic Logic, 8:370-410. 
May, R. and Wehmeier, K. F. (2016). The proof of Hume's Principle. In Ebert, P. A. and Rossberg, M., editors, Essays on Frege's Basic Laws of Arithmetic. Oxford University Press.

Schirn, M., editor (1976). Studien zu Frege. 3 vols. Friedrich Frommann Verlag, Stuttgart-Bad Cannstatt.

Schirn, M., editor (1998). The Philosophy of Mathematics Today. Clarendon Press, Oxford.

Veraart, A. (1976). Geschichte des wissenschaftlichen Nachlasses Gottlob Freges und seiner Edition. Mit einem Katalog des ursprünglichen Bestands der nachgelassenen Schriften Freges. In (Schirn, 1976), pages 49-106.

Wehmeier, K. F. and Schmidt am Busch, H.-C. (2005). The Quest for Frege's Nachlass. In Beaney, M. and Reck, E. H., editors, Critical Assessments of Leading Philosophers: Gottlob Frege, volume I, pages 54-68. Routledge, London. English translation of Wehmeier and Schmidt am Busch (2000). 'Auf der Suche nach Freges Nachlaß', in Gottfried Gabriel and Uwe Dathe (eds.), Gottlob Frege - Werk und Wirkung. Paderborn: mentis Verlag, pages 267-281.

Wright, C. (1983). Frege's Conception of Numbers as Objects. Aberdeen University Press, Aberdeen. 